\title{
DISPLASIA RENAL MULTIQUISTICA: A PROPÓSITO DE UN CASO
}

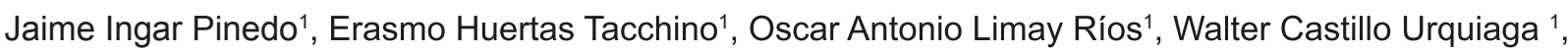 \\ Mario Zarate Girao', José Quispe Zuñiga ${ }^{2}$, Elizabeth Betteta Espejo ${ }^{3}$
}

\begin{abstract}
RESUMEN
La displasia renal multiquística es una entidad rara y poco conocida en nuestro medio. El objetivo de este trabajo es presentar y discutir en forma crítica el diagnóstico, evaluación y evolución perinatal de un caso de displasia renal multiquística, así como su diagnóstico diferencial.
\end{abstract}

Palabras clave: Displasia renal multquística; Hallazgos ecográficos (fuente: DeCS BIREME).

\section{MULTICYSTIC DYSPLASTIC KIDNEY: A CASE REPORT}

\begin{abstract}
Multicystic renal dysplasia is a rare and little known in our country. The aim of this paper is to present and critically discuss the diagnosis, assessment and perinatal outcome of a case of multicystic dysplastic kidney and its differential diagnosis.
\end{abstract}

Keywords: Renal dysplasia multquística; Ultrasound findings (source: MeSH NLM).

\section{INTRODUCCIÓN}

La displasia renal multiquística (DRM) es la presencia de riñones poco desarrollados con diferenciación pobre de nefronas y tubos colectores con la presencia de formaciones quísticas, múltiples no comunicantes. Debido a que no hay comunicación con la pelvis renal, el riñón afectado es no funcionante ${ }^{1}$. Su prevalencia varía de $1 / 2000$ a $1 / 7000$ nacidos vivos (1/4000 promedio), siendounilateral en $75 \%$ de los casos y predominando en varones $(60 \%)$; sin embargo, la forma bilateral predomina en mujeres. El $25 \%$ de los casos está asociado a otras malformaciones y el $10 \%$ a aneuploidias ${ }^{2}$.

Su fisiopatología radica en una obstrucción temprana del sistema colector embrionario, produciendo una inadecuada interacción entre el mesénquima y tejido colector primitivo conllevando a una proliferación excesiva del uroepitelio(quistes) o apoptosis del mesénquima(atrofia). Dentro de los genes involucrados se encuentra la mutación del gen TCF2, sobreexpresión del PAX2 y uroplaquinas ${ }^{3}$.

A nivel macroscópico se evidencian riñones grandes multiquisticos y un uréter no permeable y obstruido; a la microscopía tejido urotelial y glomérulos no organizados, escasos, con hipertrofia del estroma y en algunos casos presencia de cartílagos ${ }^{4}$.

Dentro de los hallazgos ecográficos se encuentran riñones grandes hiperecogenicos con quistes corticales simples de paredes delgadas no interconectados, el borde renal es irregular, parénquima hiperecogénico, pelvis renal y uréter no evidente y el flujo doppler renal es disminuido o ausente; en el riñón contralateral se observa dilatación pielocaliceal e hipertrofia compensadora en $43 \%$ de los casos. Los hallazgos ecográficos son evidentes recién a partir de las 18 semanas $^{5}$.

El objetivo de este reporte de casos es brindar información acerca de esta patología, debido a los pocos datos existentes y al mínimo conocimiento acerca de displasia renal multiquística en nuestro medio; así como aportar bases clínicas para el enfoque diagnóstico y manejo al personal de salud.

\section{CASO CLÍNICO}

Paciente mujer de 37 años, natural de Chimbote, procedente de San Juan de Lurigancho.

Acude al Instituto Nacional Materno Perinatal (INMP), con resultado de ecografía obstétrica, donde hacen el diagnostico de gestación única de 39 semanas, con macrosomía fetal y malformación renal compatible con displasia renal. Dentro de sus antecedentes personales y familiares no existe ninguno relevante, antecedentes ginecoobstétricos, tiene 3 partos vaginales, y un aborto espontáneo en el 2008, siendo el de mayor peso de $3100 \mathrm{~g}$.

Cuenta con solo 2 controles prenatales, a las 19 semanas $\sin$ mayores alteraciones y a las 39 semanas, encontrándose una altura uterina de $39 \mathrm{~cm}$ y presión arterial $150 / 90 \mathrm{mmHg}$.

Medico Ginecólogo Obstetra de la Unidad de Medicina Fetal del Instituto Nacional Materno Perinatal. Lima Perú.

Médico Residente de Ginecología y Obstetricia del Instituto Nacional Materno Perinatal. Lima Perú.

Médico Residente de Ginecología y Obstetricia del Hospital Nacional Arzobispo Loayza. Lima Perú.

Recibido: 10-10-13 Aprobado: 3-12-13 


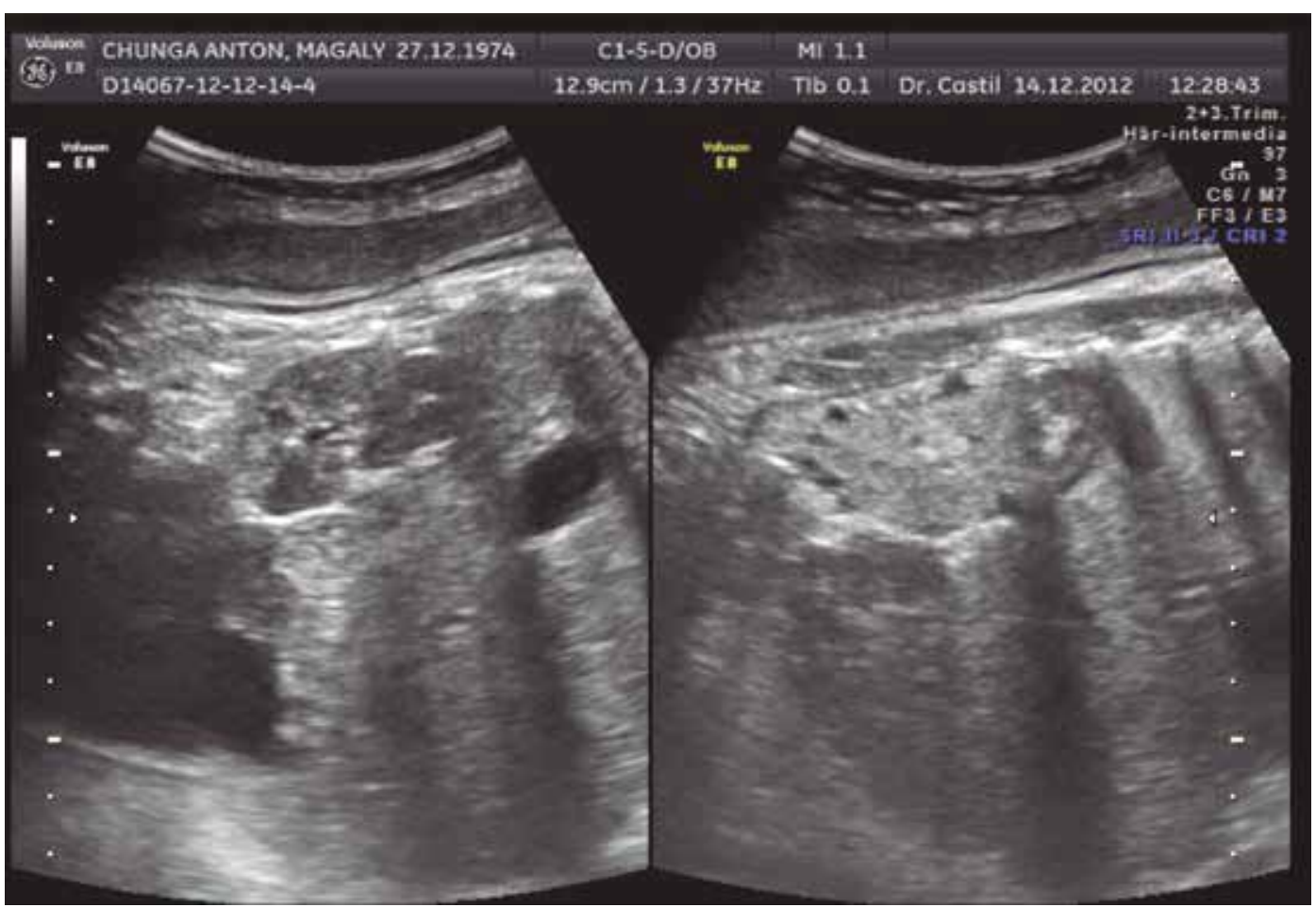

Figura 1

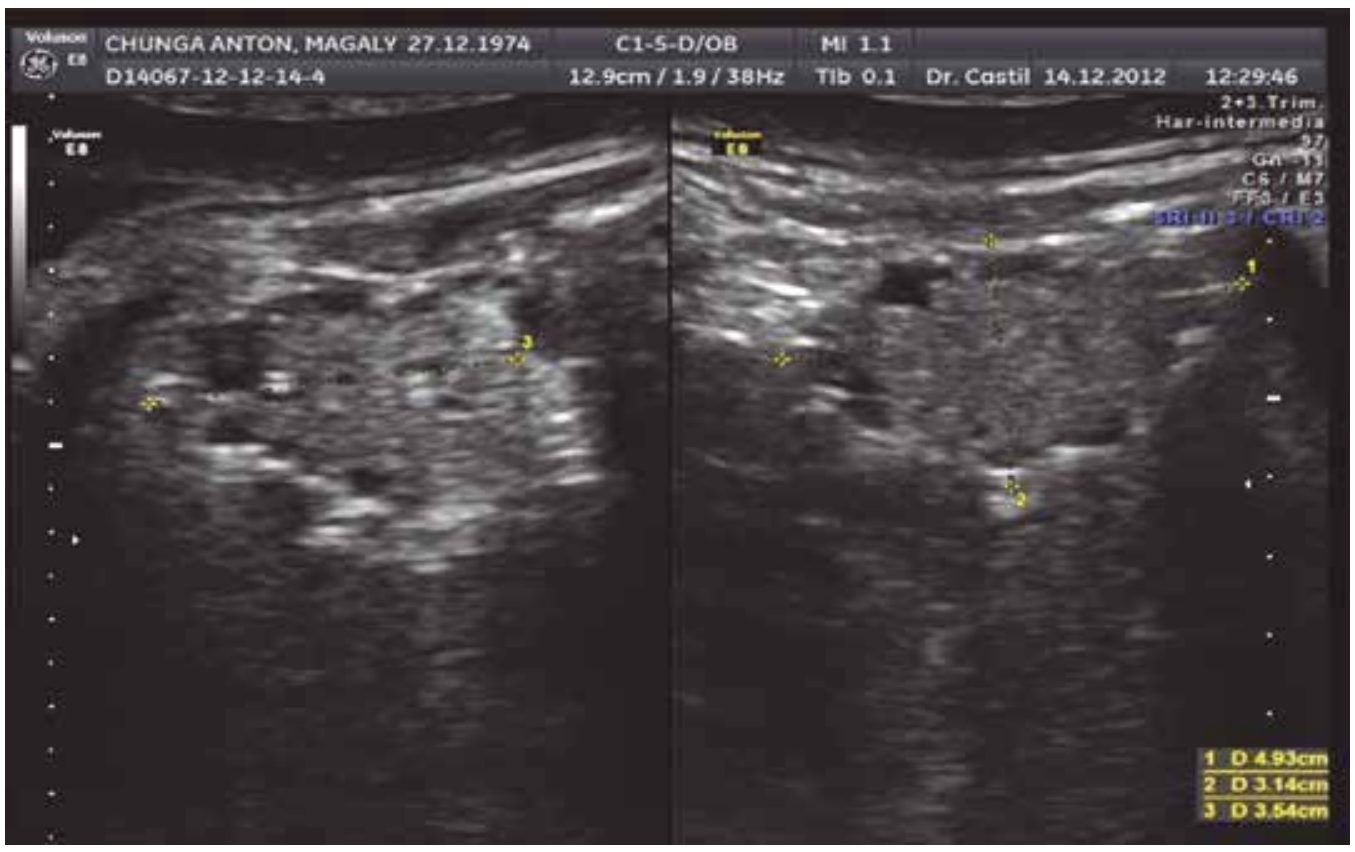

Figura 2

Figura 1 y 2. Ultrasonografía fetal donde se muestran all riñon izquierdo y derecho respectivamente, donde se evidencia un riñon derecho hiperecogénico en relación al izquierdo y a la vez la presencia de múltiples quistes corticales.

Demuestra una ecografía obstétrica a las 17 semanas, donde no evidencian malformaciones fetales.

En la ecografía que se realiza en la INMP, en diciembre del 2012, evidencian una gestación de 39 semanas por biometría fetal, una malformación renal derecha compatible con displasia renal multiquistica con diámetros renales de $49 \times 31 \times 25 \mathrm{~mm}$, más hepatomegalia por perímetro abdominal, doppler de arteria umbilical normal, perfil biofísico 8/8, macrosomía fetal, con un peso de 4563 $\mathrm{g}$ ( Figura 1 y 2 ).

Al sexto día de hospitalización, inicia trabajo de parto, por lo que es ingresada a cesárea de emergencia, con los 
diagnósticos de gestante de 40 semanas, trabajo de parto fase latente, malformaciones renales fetales, hipertensión transitoria de la gestación, macrosomia fetal, anemia leve. Se obtiene un recién nacido de sexo mujer; peso $4504 \mathrm{~g}$; talla $51 \mathrm{~cm}$.; Apgar: 9 al minuto y a los 5 minutos. El líquido amniótico fue meconial espeso, con índice de líquido amniótico normal y no había mal olor. Por lo que al diagnóstico final se le adiciona sufrimiento fetal agudo. En los estudios de pre eclampsia que se hicieron, los análisis de laboratorio resultaron dentro de los parámetros normales. Al neonato le realizan una ecografía abdominal donde concluyen que el abdomen superior es de aspecto normal, el hígado y bazo se encuentran dentro de los parámetros normales y se encuentra un riñón derecho displásico.

Durante su estancia, la paciente y el neonato cursan con evolución favorable, por lo que son dados de alta al tercer día post operatorio.

\section{DISCUSIÓN}

La DRM es un entidad rara y su diagnóstico diferencial incluye una serie de enfermedades renales quísticas ${ }^{4}$. El hallazgo ecográfico clásico esla presencia de riñones grandes hiperecogénicos con quistes corticales simples no interconectados; los cuales varían en tamaño pudiendo ser gigantes ${ }^{6}$. Asimismo su presentación puede ser bilateral $(25 \% \text { casos })^{7}$ o incluso ser segmentaria afectando en su mayoría de veces el polo renal inferior del lado afecto $^{8}$. Muchas veces se presentan como quistes gigantes simples únicos diagnosticados luego del estudio anatomopatológico ${ }^{9}$. El diagnóstico prenatal se basa en el uso de ecografía obstétrica 2D, pudiendo ser la ecografía 3D una herramienta auxiliar en el diagnóstico de la misma ${ }^{10}$ y la resonancia magnética fetal nos ayuda a diferenciar si existe un patrón obstructivo. En el lado no afecto puede observarse una dilatación pielocaliceal transitoria asociado a una hipertrofia compensadora (43\% casos), el cual puede evaluarse midiendo el diámetro renal antero-posterior entre el transverso siendo indicativo de hiperplasia el valor mayor de 1 en el segundo y tercer trimestre ${ }^{11}$.

Durante el examen ecográfico de los tumores renales debe evaluarse el tamaño renal (debajo percentil 5 o encima percentil 95) $)^{12}$, ecogenicidad renal, presencia de quistes, el líquido amniótico y la presencia de anomalías asociadas ${ }^{1}$.

Dentro de las anomalías contralaterales asociadas en mayor frecuencia figura el reflujo vesico-ureteral(RVU) $(20 \%)$ y el segundo la estenosis de la unión pieloureteral(5\%). En los pacientes con RVU el $60 \%$ es leve y $40 \%$ es severo ${ }^{17,18}$.

El manejo de los pacientes con DRM a nivel prenatal es conservador, realizando ecografías seriadas para monitorizar el líquido amniótico y la presencia de lesiones contralaterales ${ }^{1}$.

El manejo post natal ha variado en años recientes debido al conocimiento de la evolución de la enfermedad. Se sabe que le DRM unilateral tiene un buen pronóstico dado que el $33 \%, 47 \%$ y $59 \%$ involuciona a los 2,5 y 10 años respectivamente ${ }^{13}$, teniendo como predictor el tamaño renal $<62 \mathrm{~mm}$ para involución ${ }^{19}$; además el riesgo de desarrollar hipertensión arterial es similar que el de la población general $5.4 / 1000^{14}$ y también el riesgo de cáncer renal es bajo $3.5 / 1000^{15}$. Sin embargo, a pesar que el riñón contralateral no esté afectado, existe riesgo de hiperfiltración renal $(32 \%)$ y proteinuria $(10 \%)^{16}$. De tal manera que el manejo conservador expectante se ha realizado en varios centros hospitalarios en más del $85 \%$ de los casos con buenos resultados perinatale ${ }^{20}$. En dicho manejo se realiza ecografía post natal para confirmar diagnóstico, seguimiento y evaluar afección contralateral (C/6meses), estudio nuclear de función renal, exámenes de función renal, examen de orina y toma de presión arterial; recomendándose un seguimiento no menor de 30 meses $^{21}$.

El tratamiento quirúrgico es reservado para casos de hipertensión arterial no controlada, aumento progresivo de volumen tumoral y sospecha de malignidad, siendo este realizado en un $5 \%$, consistiendo en nefrectomía ${ }^{22}$ (Figura 2).

El pronóstico y diagnóstico diferencial depende ampliamente del tipo de lesión, el compromiso bilateral y la presencia o no de enfermedades genéticas o aneuploidias asociadas $^{14}$.

\section{CONCLUSIÓN}

La DRM es una enfermedad rara con poca asociación genética; generalmente es unilateral a predominio levemente masculinoy diagnosticado a partir del segundo trimestre. La evolución clínica es favorable en la mayoría de casos, con regresión del $60 \%$ de los casos. El Manejo pre y post natal más recomendado es expectante, con monitoreo semestral hasta los 30 meses de edad, por lo que pocos pacientes son sometidos a nefrectomía actualmente.

\section{REFERENCIAS BIBLIOGRÁFICAS}

1. Copes, Gratacos, et al. Obstetric Imaging. Editorial Elsevier 2012: Pag.:63 - 67

2. Van Eijk et al. Unilateral multicystic dysplastic kidney: a combined pre- and postnatal assessment. Ultrasound Obstet Gynecol 2002; 19: 180-183

3. Winyard C. Dysplastic kidneys.Seminars in Fetal and Neonatal Medicine2008:13,142-151

4. Bisceglia A. Renal cystic disease. Adv Anat Pathol 2006;13:2656 
5. Wolf A. Unilateral multicystic dysplastic kidney. Kidney Inter 2006;69:190-193

6. Komura $\mathrm{K}$ et al. Antenatally diagnosed giant multicystic dysplastic kidney resected during the neonatal period. Journal of Pediatric Surgery (2008) 43, 2118-2120

7. Bault $D$. Unilateral and bilateral multicystic dysplastic kidneys in fetuses. Obst Gynecol 2007: 1225-1228.

8. Kallysousef M. Segmental multicystic dysplastic kidney in children. Urology 2006:68: 1121.e9-e11.

9. Lym t al. Multicystic Dysplastic Kidney Disease Presenting With a Single Large Cyst in a fetus: Anatomical Basis and Radiological Aspects. Pediatrics and Neonatology 2011:52; 227-231

10. Yang et al. Prenatal diagnosis of fetal multicystic dysplastic kidney in the era of three-dimensional ultrasound: 10-year experience. Taiwan J Obst Gynecol 2012; 51(4):596-602

11. Cho et al. Measurement of compensatory hyperplasia of the contralateral kidney: usefulness for differential diagnosis of fetal unilateral empty renal fossa. Ultrasound Obstet Gynecol 2009; 34: 515-520

12. Size and volume charts of the fetal kidney, renal pelvis and adrenal gland. UOG 2012

13. MCDK Study Group. Unilateral multicystic dysplastic kidney: long term outcomes. Arch Dis Child 2006;91:820-823
14. Narchi H. Risk of hypertension with multicystic kidney disease: a systematic review. Arch Dis Child 2005;90:921-924

15. Narchi H. Risk of Wilms' tumour with multicystic kidney disease: a systematic review. Arch Dis Child 2005;90: 147149

16. Mansoor N. Long-term risk of chronic kidney disease in unilateral multicystic dysplastic kidney. Pediatr Nephrol. 2011;26(4):597-603

17. Schreuder et al. Unilateral multicystic dysplastic kidney: a meta-analysis of observational studies on the incidence, associated urinary tract malformations and the contralatera kidney Nephrol Dial Transplant (2009) 24: 1810-1818.

18. Damen et al. Concomitant anomalies in 100 children with unilateral multicystic kidney. Ultrasound Obstet Gynecol 2005; 25: 384-388.

19. Rabelo et al. Predictive factors of ultrasonographic involution of prenatally detected multicystic dysplastic kidney.BJU Intern 2005;876-871

20. Ghwery et al. Multicystic Dysplastic Kidney: Conservative Management and Follow-Up. Renal Fail 2005:189-192

21. Kogan. Natural history of patients with multicystic dysplastic kidney-what followup is needed?.J Urol2006;176(4):1607-11

22. Kuwertz et al. Unilateral multicystic dysplastic kidney: experience in children. BJU Intern 2004: 388-394 